\title{
Wound Healing in the Corneal Epithelium: Biological Mechanisms and Mathematical Models
}

\author{
EAMONN A. GAFFNEY ${ }^{\mathrm{a}, *}$, PHILIP K. MAINI ${ }^{\mathrm{a}}$, JONATHAN A. SHERRATT ${ }^{\mathrm{b}}$ and PAUL D. DALE ${ }^{\mathrm{a}}$ \\ ${ }^{a}$ Centre for Mathematical Biology, Mathematical Institute, 24-29 St Giles', Oxford OX1 3LB, UK.; \\ ${ }^{b}$ Nonlinear Systems Laboratory, Mathematics Institute, University of Warwick, Coventry CV4 7AL, UK
}

\begin{abstract}
Corneal epithelium has a highly specialised wound-healing response. The biological aspects of this repair process are reviewed, and methods of modelling it mathematically are described. A model which focuses on the source of epidermal growth factor (EGF) within a healing wound is described. By considering mathematical representations of a number of possible source terms, it is shown that the EGF present in the tear film is insufficient to explain the observed rate of healing, and experimental approaches are suggested for distinguishing between other sources. Also, the simulation of exogenous addition of EGF using the model is described. An issue that has been the subject of considerable debate in the literature is the role of eyeball curvature. The model is used to show that this curvature is not significant for either the speed or form of healing in the epithelium. In conclusion, a comparison is made between wound healing in the corneal epithelium with that in the epidermis of the skin. Possible directions for future modelling work are considered.
\end{abstract}

Keywords: Biomathematical modelling, Corneal epithelium

\section{INTRODUCTION}

Complex mechanisms of wound healing have developed in mammals and other higher organisms over the course of evolution. The wound healing of the cornea is unique amongst such mechanisms, because of the overwhelming need to maintain visual acuity. This requires that non-pathological corneal wound healing cannot, for example, entail vascular invasion of the wound. Hence, corneal wound healing is both relatively simple and of key medical importance, and this is reflected by a vast literature on the subject, much of which has recently been quantitative. The emergence of such data provides many opportunities for mathematical modelling to play a key role in research development.

Here we will consider a specific aspect of corneal repair, namely epithelial corneal wound healing (ECWH). We explicitly discount the pathological possibility of vascular ingrowth (angiogenesis) and only make the briefest mention of conjunctival in growth onto the cornea. There is a similar neglect of the role of innervation, the immunological system and many other biological phenomena. We thus, simplistically, consider a system of epithelial cells (in various states of differentiation), and a basement lamina consisting of a variety of extracellular matrix (ECM) components. The

\footnotetext{
* Conresponding Author: Tel. 01865 280601; Fax: 01865 273583; E-mail: gaffney@maths.ox.ac.uk.
} 
tissue dynamics are controlled by numerous types of cell-cell and cell-substrate interactions. These interactions, together with the cell proliferation and differentiation rates and ECM component synthesis rates, are critically dependent on dozens of different growth factors. These growth factors, often referred to as cytokines, originate from numerous possible sources including cell excretions, tear fluid, and in a wounded cornea, cell debris and possibly exogenous application.

Specific aspects of such a simplified system can provide an initial framework, based upon recent advances in the modelling of cellular populations, capable of encapturing, interpreting and summarising much of the recent quantitative experimental data. It should also be able to contribute to the understanding of how cellular level activities of the epithelial cornea affect its macroscopic behaviour and highlight areas requiring further investigation.

A moderately detailed account is given of the corneal epithelium and its wound healing response. We proceed to identify some key questions concerning ECWH which are can be addressed using tractable mathematical models. Then we expand on the discussion of growth factors, especially with respect to the controversial question of their source. Previous modelling work which addressed this is summarised, and the possibility of auto-inductive growth factor production is also discussed. We discuss the importance, or lack thereof, of eyeball curvature for ECWH modelling and corneal wound healing modelling in general. Finally, a comparison is made between the epithelial corneal woundhealing response and other wound-healing scenarios which have been modelled mathematically, such as repair in adult and embryonic epidermis.

\section{ECWH IN DETAIL}

There are many excellent reviews of the biology of the cornea. One particularly recommended selfcontained review, assuming minimal background, is contained in the book by Forrester et al. [14]. More specialised and detailed reviews can be found in the references $[15,18,29]$.

\section{The Healthy Corneal Epithelium}

\section{The tear film}

The surface of the cornea is coated with a tear film, which acts as a protective cover. It also supplies nutrients to the corneal epithelial cell layer which it overlies and is known to contain growth factors including epidermal growth factor (EGF), which will be discussed in detail below.

\section{The corneal epithelium and limbus}

The corneal epithelium is a transparent stratified layer, approximately six cells thick [14], of interlinked cells, acting as a protective cover for the surface of the eye. The upper layer of these cells are the squamous epithelial cornea (EC) cells which are terminally differentiated, and continually shed into the tear film, to be renewed from below. To replace these cells, there is continual cell proliferation even in the unwounded cornea. Beneath the squamous layer, are the post-mitotic epithelial wing cells, named after their shape. Below these is the basal layer of epithelial cells, which are capable of proliferation. This cell layer is attached to a basement lamina whose major constituents are, in the unwounded cornea, fine collagen fibres $(20-30 \mathrm{~nm}$ diameter, mainly type IV collagen [29]), laminin and proteoglycans. Fibronectin only appears in significant quantities in the basement lamina of a wounded cornea [29], as detailed below.

In the unwounded cornea, the cell-cell interactions occur via cadherin based cell adhesions and desmosome type cell junctions[16,45]. These references also give details of hemidesmosomes, which govern the adhesion of the basal layer of epithelial cells to the underlying basement lamina in the unwounded cornea.

The transitional area between the transparent tissue of the cornea and the opaque tissue of the conjunctiva and sclera surrounding the cornea is referred to as the limbus. The limbus, as discussed below, plays a crucial role in epithelial biology. 
Origim, migration, proliferation and differentiation of epithelial cells

The origin of the epithelial cells, together with their differentiation, migration, and proliferation has been the subject of extensive study. Regarding the origin of the epithelial cells, the status quo is summarised in the The Limbal Stem Cell Hypothesis [22]. This states that, located in the basement layer of the limbus surrounding the corneal epithelium, there are epithelial stem cells (described below), responsible for the self-renewing properties of the corneal epithellium.

Sterh cells have unique properties [42]. They are (relatively) undifferentiated, have long cell cycle times and low mitotic rates. However, they are long-lived with an almost unlimited potential for (virtually) error-free clonogenic division. They are also capable of producing, via two distinct mechanisms [42], transient amplifying cells. The first of the two mechanisms is asymmetric cell division, produding two daughter cells, one of which is a stem cell and the other a transient amplifying cell. The seicond mechanism results in the stem cell differentiating to form a transient amplifying cell. The details of these mechanisms are unclear [42]. The transient amplifying cells are short lived, but with high mitotic rates and are responsible for virtually all of the cell proliferation in the cornea and limbus.

The resulting differentiation, proliferation and migration of these basement layer transient amplifying cellls has been studied $[42,20,16]$. There is strong evidence that these cells migrate radially inwards and uptwards away from the limbal basement region. An individual transient amplifying cell will migrate along the basement layer for a given distance before proliferating, whereupon one daughter cell is displaced into the wing cell layer and the daughter cell takes the mother cell's place on the basement lamina [16]. A basal cell displaced from the basement lamina will differentiate to the post-mitotic wing cell form. This displaced and subsequently differentiated cell may move further inward, before being displaded into the squamous layer, whereupon it terminally differentiates into the squamous cell form. From here, it may again migrate inwards, before being shed into the tear film. The radial component of the above migration must be inhibited near the centre of the cornea, possibly by contact guidance mechanisms. It takes approximately 5-7 days between displacement from the basement lamina and shedding into the tear film for a typical cell [16].

The nature of this migration, which is subject to standard conservation laws, is described in the biological literature as the XYZ hypothesis [41]. This hypothesis has motivated simple models of epithelial cell maintenance [34].

\section{The Epithelial Corneal Wound Healing Response}

ECWH is a complicated multi-phase process [29]. For simplicity, we concentrate mainly on the scenario in which the basement lamina has remained intact, but there has been removal of epithelial cornea. This results in a pre-migratory phase, during which time the exposure of the basement lamina to tear fluid and cell debris results in the expression of fibronectin in the denuded wound bed. Simultaneously, the surrounding basal cells disassemble their cell-substrate contacts and increase their expression of fibronectin receptors (a type of integrin $[29,45]$ ) in preparation for migrating across the wound bed. Rearrangement of the cell-cell adhesions and junctions must also occur, although some desmosomes remain intact. The detailed changes in cell-cell interactions when a cornea is wounded are not fully understood [16].

In the migratory phase, a monolayer of basement epithelial cells spreads across the wound bed, undergoing a cycle of forming and breaking focal contacts with the fibronectin expressed in the wound bed $[16,45]$. Fibronectin mediated haptotaxis occurs in vitro [23] and may play an important role in vivo as well. Following behind, and on top of, this migrating monolayer is a proliferation driven multi-layer 'landslide' of migrating and undifferentiated epithelial cells. In practice, a significant proportion of the wound bed is initially covered by only the migrating monolayer [12]. Subsequently, the lagging multilayer proceeds to move over this 
initial basal monolayer, thus filling the epithelial defect.

The final phase consists of differentiation of the cells covering the wound bed, so that the epithelial cornea regains its unwounded stratified structure. It also consists of the reformation of hemidesmosomes adhering the basal cells to the basement lamina.

\section{Further Aspects of ECWH}

Growth factors, a genetic term describing a ubiquitous class of chemicals regulating cell growth and proliferation, play an important role in the above framework. In the unwounded cornea, they regulate the balance between cell loss and proliferation. In the wounded cornea they control the response to wounding, via exocrine, autocrine and paracrine pathways.

Epidermal growth factor (EGF), for example, is a powerful stimulator of epithelial cell proliferation [44]. There is also strong evidence that it increases the expression of fibronectin receptors [23] in epithelial cells that stimulates in vitro [3] and in vivo [29] migration of epithelial cells. This migratory stimulus is down regulated, being suppressed at sufficiently high concentrations of EGF. There have been many reports on the efficiency of EGF in the treatment of clinical disorders [29], together with evidence that it does not induce serious complications such as corneal vascularisation [32]. Many other growth factors have also be implicated in repair of the corneal epithelium (see [15] for a summary), and, in particular, transforming growth factor- $\beta$ plays an important role in regulating the effects of EGF.

Despite a worldwide program of experimental research, there remains considerable uncertainty in the literature regarding the details of the qualitative and quantitative effects of the growth factors on epithelial cells. For example, there is conflicting evidence on the chemotactic and chemokinetic effects of EGF on epithelial cell movement $[15,17,23]$. It is also unclear whether gradients of growth factors, which are crucial for a chemotactic response, can be generated within a corneal wound. Another important controversy concerns the source of growth factors in an epithelial corneal wound. This has been addressed in our previous modelling work [7,8], which is discussed in detail below.

Finally on the subject of chemical stimuli, there is evidence that the addition of fibronectin and hyaluronic acid stimulate migration synergistically in vivo, as deduced by Nakamura et al. [28] This might be expected as Nakamura's previous work [27], based on in vitro cultures, had indicated that fibronectin has a binding site for hyaluronic acid and that the binding between hyaluronic acid and fibronectin facilitated the latter's attachment, via focal contacts, to epithelial cells. In turn, provided there is sufficient protease activity to enable these focal contacts to be disassembled as required throughout the path of the migrating cells, this could increase migration rates. The general issue of synergism in growth factor activity in the cornea is discussed by Schultz et al. [33].

ECWH has an important role to play in the initial healing of radial keratectomy wounds. These are incisions relatively deep into the cornea, whose aim is to change the refractive properties of the cornea. Here the epithelium migrates into the keratectomy wound and the subsequent mass landslide forms an epithelial plug. The fact that basement lamina is pierced leads to a more extensive interaction of the basement layer with the underlying corneal layers (the stroma), resulting [18] in an increased expression of epithelial components, such as fibronectin. Thus post-keratectomy ECWH offers a key alternative application of mathematical modelling of the cornea, and will also be discussed in more detail below.

Another important potential application of mathematical modelling is to limbal grafting, a process whereby a proportion of the limbus from a donor comea is grafted onto a recipient cornea. This is performed in response to some limbal failure in the recipient cornea in an attempt to maintain and/or restore recipient corneal integrity. The donor cornea may sometimes, in unilateral disorders, be the patient's asymptomatic, apposing cornea. Heterogeneity is an integral part of the ECWH of 
both the donor and the recipient cornea after grafting and would be a key feature in any model attempting to investigate the ECWH associated with limbal grafting.

Finally in this section, we mention speculation in a recent paper [10] on the possible role of small ocular eledtromagnetic fields on the in vivo behaviour of epithelial cells. The authors of this paper noted a spiral pattern upon numerous patients' corneas with $1 \%$ Sodium fluorescein. The pattern was independent of the precise nature of the patients' corneal disorders and thus was speculated to be the result of proliferatory and migratory properties of the corneal cells. It was further speculated that small ocular electromagnetic flelds could be responsible for the pattern and that these fields might perturb the migration paths of epithelial cells across the corneal. The exact mechanism for the spiral stain pattern remains unclear.

\section{GROWTH FACTORS IN EPITHELIAL REPAIR}

As described above, it is generally accepted that epithelial corneal wound healing is strongly controlled by EGF, but many details of this regulation remain unclear. In this section, we discuss mathematical modelling which addresses two of these details: the relative contributions of EGF to cell migration and mitosis, and the dominant source of EGF. Our presentation reviews and extends the work $\phi$ f Dale et al. [7,8].

Our model consists of conservation equations for cell density, $n(x, t)$, and extracellular EGF concentration, $c(x, t)$. A more realistic model would be much more complex, including variables representing extracellular matrix elements and their possible haptotactic effects [29,23], and transforming growth factor- $\beta$, which regulates the EGF response of corneal epithelial cells [15]. However, we deliberately neglect these factors, in order to concentrate on the details of the production of, and cellular response to, EGF. With the same motivation, we also neglect the threedimensional structure of the epithelium and its underlying basal lamina, representing the epithelium as a two-dimensional sheet. This can be justified on the basis that typical wound diameters (several millimeters) are very much larger than the thickness of the epithelium (about $60 \mu \mathrm{m}$ ). However, the three-dimensional structure may play a role in the repair process, and this is another aspect that would be important to include in an improved model.

Our model equations have the following qualitative form:

Rate of change of cell density

$=$ Cell movement + Cell division - Cell loss

Rate of change of EGF concentration

$$
\begin{aligned}
= & \text { EGF diffusion }+ \text { Production } \\
& - \text { Removal and decay }
\end{aligned}
$$

Since our main interest is in the production of EGF and its effects on cell migration and division, we use very simple, generic representations of the other terms in the model. Specifically, we assume a linear rate of natural cell loss, $-k n$; the constant $k$ is quite large because the flow of the tear film across the epithelial surface ensures a rapid natural turnover of cells. In comparison, the loss of epidermal cells from the skin surface is much slower [30]. For chemical diffusion, we simply take the standard Fickian form $D_{c} \nabla^{2} c$. Chemical decay occurs via two mechanisms: natural decay, with a half-life of about an hour [4], and cell-induced decay via binding and bound receptor internalisation at the cell surface. Standard arguments [7] show that an appropriate term for the latter decay rate is $\mu n c /(\hat{c}+c)$, where $\mu$ and $\hat{c}$ are positive constants related to the binding kinetics of EGF to its cell surface receptor.

The terms representing cell migration and division require more detailed discussion. Both of these processes are upregulated by EGF, which stimulates DNA synthesis [2] and fibronectin adhesion [29]. We assume that both processes can be increased by about an order of magnitude by EGF stimulation, and we represent cell movement as $\nabla \cdot\left[D_{n}(c) \nabla n\right]$, and cell division as $s(c) n\left(v-n / n_{0}\right)$. Here $D_{n}($. and $s($.$) are increasing functions, and v$ and $n_{0}$ are positive constants; $n_{0}$ denotes the unwounded cell 
density. To be specific, we model cell migration by linear diffusion, with the diffusion coefficient dependent on EGF concentration, and we use a logistic term to represent cell division, in which the growth rate increases with the local EGF concentration.

There is no general agreement on the source of EGF in the wound environment. Tear fluid contains a known level of EGF (about $4 \mathrm{ng} \mathrm{m}^{-1}$ [31]), and cells within the wound will certainly be exposed to this growth factor. The basic controversy is whether this accounts for all the EGF present in the wound, or whether there is an additional growth factor release in response to wounding, either from the underlying stroma or by epithelial cells, in an autocrine manner. EGF release by the stroma is suggested by standard patterns of in vitro healing when an underlying growth factor source is simulated experimentally and autocrine production of EGF by corneal epithelial cells has been demonstrated in vivo [17]. To consider these various possibilities, we use three different forms for the production rate $\sigma(n)$ of GF:

(a) $\sigma(n)=A_{0}$, a constant. This represents EGF production by the tear film only, with no additional sources.

(b) $\sigma(n)=A_{0}+B(n)$, where $B($.$) is a decreas-$ ing function, equal to zero at the unwounded level of cell density. This represents EGF release by underlying stroma, which we assume to decrease to zero as the epithelial sheet approaches confluency. To be specific, we use the piecewise linear form

$$
B(n)= \begin{cases}B_{0} & \text { if } n<0.2 \\ B_{0}(2-5 n) & \text { if } 0.2<n<0.4 \\ 0 \text { if } n>0.4 & \end{cases}
$$

where $\sigma$ is a positive constant.

(c) $\sigma(n)=A_{0}+n G(n)$, where $G($.) is the rate of autocrine production of EGF per cell, which we assume to decrease with cell density due to feedback effects. To be specific, we use the functional form

$$
G(n)=G_{0} /\left(G_{1}+n^{2}\right)
$$

where $G_{0}$ and $G_{1}$ are positive constants.

The particular forms used for $B($.$) and G($.$) are$ not significant; the key difference is that $G(n)$ is multiplied by $n$ in the production term, so that there is no production in the absence of epithelial cells.

Thus the overall mathematical model is

$$
\begin{aligned}
\partial n / \partial t= & \nabla \cdot\left[D_{n}(c) \nabla n\right]+s(c) n \\
& \times\left(\nu-n / n_{0}\right)-k n \\
\partial c / \partial t= & D_{c} \nabla^{2} c+\sigma(n)-\mu n c /(\hat{c}+c)-\lambda c(1 b)
\end{aligned}
$$

The appropriate parameter values have been mentioned briefly above, and are discussed in more detail elsewhere [7]. An important aspect of the corneal epithelial system is the relative abundance of quantitative data from in vitro and in vivo experiments, which enables parameters to be accurately estimated.

In Figure 1, we show simulations of the healing of a circular wound, $6 \mathrm{~mm}$ in diameter, using each of the three possible production terms. We plot the evolution of the cell density and chemical concentration for the 37 hours after wounding; this is the healing time that is observed for such a wound in vivo in the rabbit, which is the best studied experimental model [6]. Details of boundary and initial conditions are given in the figure legend.

The key result of simulations such as those illustrated in Figure 1 is that the known level of EGF in the tear film is much less than would be necessary for the wound to heal in the experimentally observed time. This is a robust result, not sensitive to small parameter variations, and thus we conclude that there must be an additional source of EGF in the wound. In contrast, both the stromal and autocrine source terms (Figures $1 \mathrm{~b}$ and $1 \mathrm{c}$, respectively) do predict healing in the experimentally observed time. The exact value of the healing time is dependent on the parameters in $B(n)$ and $G(n)$, for which we only have order of magnitude estimates, and in the figure we have chosen particular values to give an exact match with the experimental healing time. The key point is that such an exact match is possible with experimentally realistic parameter values.

Since both the stromal and autocrine source terms give appropriate healing times, we must consider whether our model is able to predict another way of distinguishing between them, on the basis of 

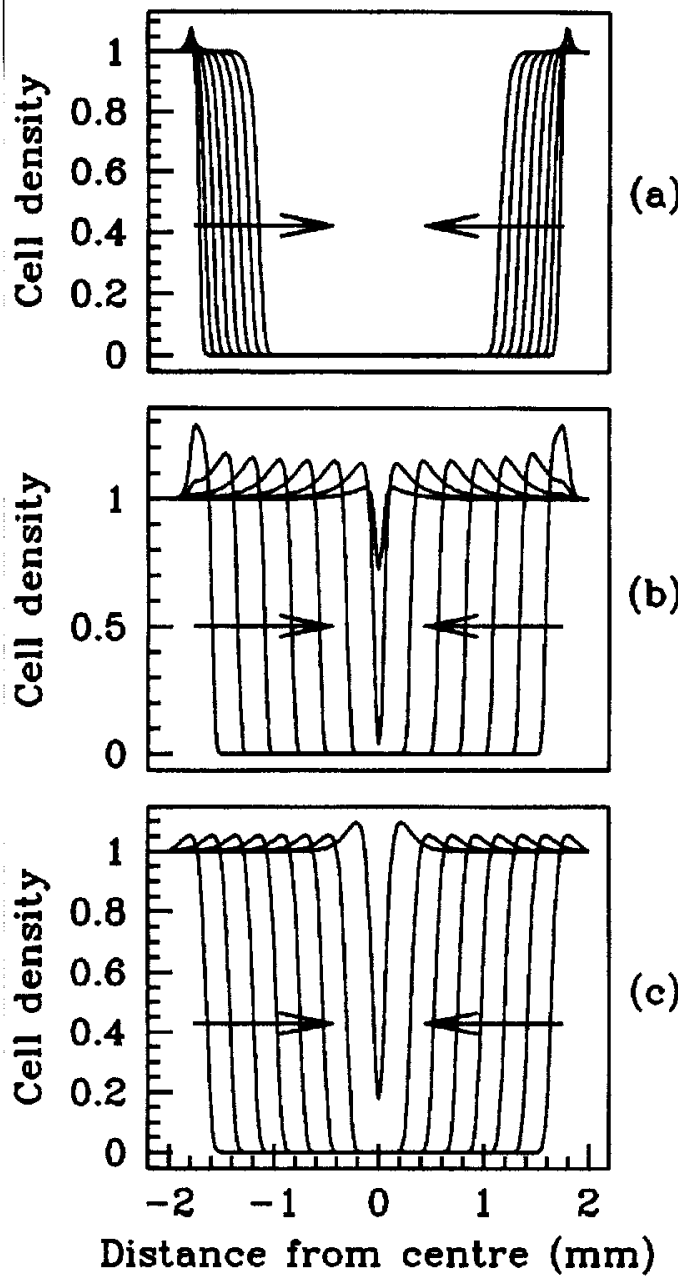

(a)

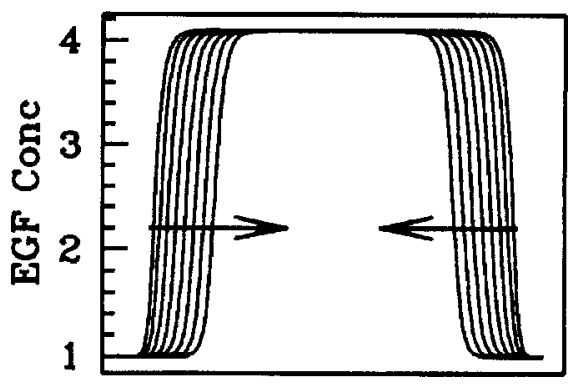

(b)
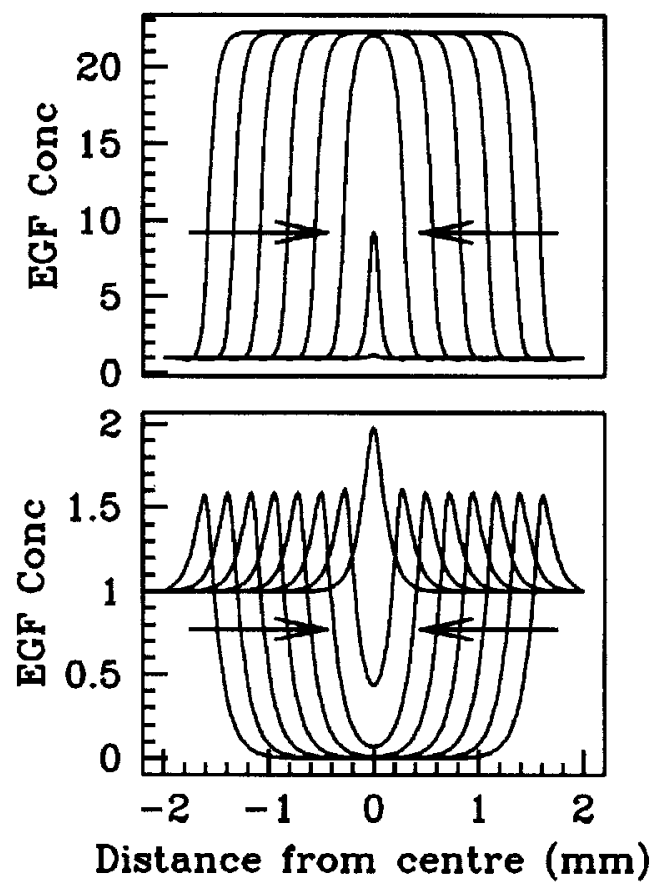

FIGURE 1 Simulation of the healing of a 4-mm diameter corneal epithelial wound, using equations (1), for the three different sources of EGF discussed in the text. Cell density and EGF concentration are plotted, as a proportion of their unwounded levels, as functions of space along a diameter of the wound, at equal time intervals over a 37-hour period. In case (a) the source term reflects EGF supply by the tear film only; case (b) corresponds to additional supply from the underlying stroma; and case (c) represents additional autocrind production by the epithelial cells. In (b) and (c), the simulated wound heals in the experimentally observed time, but in (a) it does not. The simulations are obtained by numerically solving a dimensionless version of (1), obtained via parameter estimation and non-dimensionalisation; for details, see $[7,8,35]$.

macrosicopic experimental data. The solutions for EGF concentration $c(x, t)$ in Figure 1 show that the obviou $\$$ approach for this is careful measurement of the EGF profile during healing. If the growth factor is released by the underlying tissue, then there is a high level in the centre of the wound during healing, since production rates will be highest when cell density is low, that is, in the centre of the wound. Conversely, in the autocrine mechanism, EGF concentration is very low in the centre of the wound, ahead of the repopulating wave of epithelial cells. Here, the maximum EGF concentration occurs in the wound front itself, corresponding to intermediate levels of cell density.

There is strong experimental evidence that EGF upregulates both cell movement and cell division. However, the relative contributions of these two factors to wound repair is less clear. Our model enables this issue to be addressed, since we can artificially remove the EGF dependence in either the 
cell movement or mitosis terms in the cell conservation equation. Simulations of these amended models give very similar results with either the stromal and autocrine source terms. Removal of EGF effects on cell migration does not alter the qualitative form of the predicted healing profile; the speed simply decreases (at a rate approximately proportional to the square root of the cell dispersal rate $[7,8]$ ). In contrast, removing the dependence on EGF concentration of cell division is more significant: in particular, the characteristic post-wounding lag followed by a linear rate of healing is lost.

\section{EYEBALL CURVATURE AND CORNEAL EPITHELIAL REPAIR}

It has been suggested that current analysis of corneal epithelial wound closure is misleading and inaccurate due to the simplicity of the models, particularly with respect to the validity of using planar kinematics to model the curved surface of the cornea [19]. The model formulation above can be used to examine the role of curvature for the simple case in which the eyeball is considered as a sphere. Letting the origin of the $(r, \theta, \phi)$ coordinate system be the centre of the eyeball and $r$ the radius of the eyeball, we assume that solutions are independent of $r$ and the azimuthal angle $\phi$. Equations (1) become

$$
\begin{aligned}
\frac{\partial n}{\partial t}= & \frac{1}{R} \frac{\partial}{\partial \theta}\left(\frac{1}{R \sin \theta} \frac{\partial}{\partial \theta}\left(D_{n}(c) n \sin \theta\right)\right) \\
& +s(c) n\left(v-n / \mathrm{n}_{0}\right)-k n \\
\frac{\partial c}{\partial t}= & \frac{D_{c}}{R^{2}}\left(\frac{\partial^{2} c}{\partial^{2} \theta}+\frac{1}{\theta} \frac{\partial c}{\partial \theta}\right) \sigma(n)-\mu n c /(\hat{c}+c)-\lambda c
\end{aligned}
$$

where $R$ is the radius of the eyeball. Numerical simulations of this model show that, after an initial rapid increase, the speed of the wave increases monotonically as $\theta$ increases but, crucially, for biologically realistic parameters, this increase is very small. These numerical results are confirmed by analytical calculation of the wavespeed (see [9] for full details).

\section{DISCUSSION}

Having considered the important features of ECWH some key areas and questions for modelling investigation are summarised below.

\section{Key Areas and Questions for Mathematical Modelling}

\section{Cytokines}

Clearly there is much scope for detailed modelling aimed at encapturing the role of growth factors in ECWH. One could, for example, develop models with chemotactic or chemokinetic cell density diffusion terms, or perhaps even both. An aim of such modelling would be in the design of alternative in vitro experiments to distinguish between possible cell-EGF interaction mechanisms. Models could also be developed which incorporate more than one cytokine and which encapture possible synergistic interactions, with the aim of summarising, interpolating and perhaps even extrapolating existing data.

\section{Keratectomy}

There is a vast amount of data on wound healing after keratectomy which would motivate modelling this aspect of ECWH. The first task which would have to be addressed in such modelling is the effect of different eye shapes. Although the constant curvature of the normal ocular surface does not effect existing models of ECWH, the rapidly changing curvature in the vicinity of a keratectomy wound might. However, one could model such curvature by a few extra terms in the equations. This is completely analogous to the fact that changing form a 'plane' eye to a spherical or even an ellipsoidal eye merely introduces 'curvature terms' in the equations. Such terms can be quickly deduced for an arbitrary surface by appeal to simple differential geometry.

The critical aspect of post-keratectomy ECWH is the effect of the increased epithelial cell-substrate interaction, as referred to above. An aim could be to encapture the form of this interaction via hypotheses 
deduçd from the biology of migrating corneal epithelia. Given the limited differences between normal and post-keratectomy ECWH, the data from both of these scenarios would provide a unique test of such epithelial cell-substrate hypotheses. This, in turn, would provide valuable insight into cell-substrate interaction modelling and could also be incorporated into some of the more detailed possible ECWH modelling schemes mentioned below.

\section{Heterdgeneity}

A key area for development is the modelling of ECWH with spatially heterogeneous initial conditions. The pattern formation of such repair is critically regulated by contact inhibition [11] in the vicinity of colliding wave fronts of migrating epithelial on the wound bed. A crucial question is whether tractable models can encapture the observed healing patterns and healing rates in this more complicated scenario. Heterogeneous modelling techniques could also be used to investigate the hypothesised effects of small electromagnetic fields on cell migration and perhaps to test simple hypotheses regarding the formation of the spiral staining pattern.

\section{Limbal effects on ECWH}

A major goal for future development is to encapture the effects of a finite stem cell reserve in models of ECWH. For example, work is in progress on 1D models which encapture plausible stem cell dynamics within ECWH. On completion these simple models could be compared to experiments with repeated wounding [21], designed to directly test for a finite stem cell reserve. Generalising such modelling to include substrate, heterogeneity and conjunctival effects is vital for investigating important, dlinically relevant, issues in ECWH such as limbal grafting. Within this context, such detailed ECWH modelling could address a multitude of questions such as "how much limbus needs to be grafted onto a recipient eye to regenerate a stable, injury resistant, corneal epithelium?' Attempting to embark on such a program will require extensive cooperation with biomedical colleagues and brings us to arguably the most important, but most tentative, aim of ECWH modelling. This is namely an investigation into the extent to which sophisticated modelling can increase the efficiency of the experimental process for ECWH.

\section{Other epithelial wound healing systems}

The wound-healing response of corneal epithelium is significantly different from that of other epithelia because of the localisation of stem cells to the limbus. Nevertheless, many of the controversies associated with corneal epithelium are also important for the healing of other epithelia. This includes epidermal skin repair, despite the fact that the epidermal migration proceeds via a different, 'rolling', mechanism [40] of cell crawling. In particular, the role of growth factor upregulation of cell motility and cell division is a ubiquitous controversy. In the cornea, EGF is thought to be the main chemical mediator of epithelial repair, but in the skin, no single factor has been identified as the major regulator. Indeed, it remains unclear whether upregulation of a mitotic activator or downregulation of a mitotic inhibitor is responsible for the high level of cell division post-wounding. Previous modelling work on this issue has suggested that changes in wound geometry during healing may be a feasible way of distinguishing between these mechanisms $[35,36]$.

Not all epithelia heal by cell crawling. Recently, it has been shown $[24,26]$ that wounds in the epidermis of chick and mouse embryos heal by a very different, mechanical mechanism. In these systems, a cable of filamentous act in forms within the leading layer of cells at the wound edge, as a very rapid response to wounding. This cable is continuous from cell to cell, and the wound heals by contraction of the cable, which pulls the wound edges together in a manner reminiscent of a pursestring. It remains unclear why quite different mechanisms of repair occur in adult and embryonic epidermis. The answer may lie in different growth factor 
profiles, and recent experiments have shown that the protooncogene $c$-fos and transforming growth factor- $\beta 1$ are expressed at the wound edge early in embryonic epidermal repair [25]. Mathematical modelling of embryonic epidermal repair requires quite different types of models, reflecting the complex mechanics of epithelial sheets. One important issue concerns the formation of the actin cable as a response to wounding, and modelling work by Sherratt and coworkers [37-39] has shown that this may be a relatively simple consequence of mechanical compaction at the wound edge, coupled with autoinduced alignment of act in filaments. A quite different question concerns the way in which the actin cable causes the wound to close, and this remains an open challenge for modellers.

\section{Acknowledgements}

EAG is supported by a Welcome Trust Biomathematics Post-Doctoral Training Fellowship Research Award. P.D.D. was supported by a Welcome Trust Prize Studentship. This work was supported in part by a grant from the London Mathematical Society. We thank Steven Tuft, Peng Khaw and Lorraine Waters for helpful discussions.

\section{References}

[1] Barrandon, Y. and Green, H. (1987). Cell migration is essential for sustained growth of keratinocyte colonies: the roles of transforming growth factor- $\alpha$ and epidermal growth factor. Cell, 50, 1131-1137.

[2] Bazan, H. E., Allan, G. and Bazan, N. G. (1992). Enhanced expression of the growth-regulated calcyclin gene during cornea wound healing. Exp. Eve Res. 55, 173-177.

[3] Boisjoly, H., et al. (1993). Effects of EGF. IL-1 and their combination on in vitro corneal epithelial wound healing and cell chemotaxis. Exp. Eye Res., 57, 293.

[4] Chan, K. Y., Patton, D. L. and Cosgrove, Y. T. (1988), Time-lapse videomicroscopic study of in vitro wound closure in rabbit corneal cells. Invest. Ophthalmol. Vis. Sci., 301, 2488-2498.

[5] Crosson, C. E., Klyce, S. D. and Beuerman, R. W. (1986). Epithelial wound closure in rabbit cornea. Invest. Ophthalmol. Vis. Sci., 271, 464-473.

[6] Dale, P. D., Maini, P. K. and Sherratt, J. A. (1994). Mathematical modelling corneal epithelial wound healing. Mathematical Biosciences, 124, 127

[7] Dale, P. D.. Sherratt, J. A. and Maini. P. K. (1994). On the speed of corneal epithelial wound healing. Appl. Math. Lett., $7,11-14$.
[8] Dale, P. D. (1995). Time Heals all Wounds? Mathematical Models of Epithelial and Dermal Wound Healing. D Phil thesis, Oxford.

[9] Dua, H., Watson, N., Mathur, R. and Forrester, J. (1993). Corneal epithelial cell migration in humans: 'burricane and blizzard keratopathy'. Eve, 7, 53.

[10] Dua, H. and Forrester, J. (1987). Clinical patterns of corneal epithelial wound healing. Am. J. Ophthal, 104, 481.

[11] Dua, H., Gomes, J. and Singh, A. (1994). Corneal epithelial wound healing. Br. J. Ophthal., 78, 401.

[12] Dunn, G. A. and Ireland, G. W. (1984). New evidence that growth in $3 \mathrm{~T} 3$ cell cultures is a diffusion-limited process. Nature, 312, 63-65.

[13] Forrester, J., Dick, A., McMenamin, P. and Lee, W. (1996). The Eye, W. B. Saunders Ltd, London.

[14] Gipson, I. and Inatomi, T. (1995). Extra-cellular matrix and growth factors in corneal wound healing. Curr. Opin. Ophthal., 6(4), 3.

[15] Gipson, I. (1992). Adhesive mechanisms of the corneal epithelium. Acta Ophthal., 70, Suppl. 202.

[16] Grant, M., Khaw, P., et al. (1992). Effects of EGF, FGF and TGF- $\beta$ on corneal cell chemotaxis. Investig. Ophthal. Visual Science, 33, 3292.

[17] Jain, S. and Azar, D. (1994). Extra-cellular matrix and growth factors in corneal wound healing. Curr. Opin Ophthal., 5(4), 3.

[18] Kwok, L. S. (1991). Kinematics of epithelial wound closure in the rabbit cornea. Doc. Ophthal., 77, 1.

[19] Kruse, F. (1994). Stem cells and corneal epithelial regeneration, Eye, 8, 170.

[20] Huang, A. and Tseng, S. (1988). Comeal wound healing in the absence of limbal epithelium. Investig. Ophthal. Visual Science, 29, Suppl. 190.

[21] Lavker, R., Cotsarelis, G., Dang, G., Hardy, C. Schemer, A. and Sun. T. (1988). Limbal location of corneal epithelial stem cells, in H.D. Cavanagh (ed) The Cornea, Transactions of the World Congress on the Cornea III, (Raven Press, New York), p. 23.

[22] Maldonado, B. and Furcht, L. (1995). EGF stimulates integrin mediated cell migration of cultured human epithelial cells on fibronectin and arginine-glycine-aspartic acid peptide. Investig. Ophthal. Visual Science, 35, 2120.

[23] Martin, P. and Lewis, J. (1992). Act in cables and epidermal movement in embryonic wound healing. Nature, $\mathbf{3 6 0}$, $179-183$.

[24] Martin, P., Nobes, C., McCluskey, J. and Lewis, J. (1994) Repair of excisional wounds in the embryo. Eye, 8, $155-160$.

[25] McCluskey, J., Hopkinson-Woolley, J., Luke, B. and Martin, P. (1993). A study of wound healing in the E11.5 mouse embryo by light and electron microscopy. Tissue and Cell, 25, 173-181.

[26] Nakamura, M., Mishima. H., Nishida, T. and Otori (1994) Binding of hyaluronic acid to plasma fibronectin increases the attachment of corneal epithelial cells to a fibronectin matrix. J. Cellular Physiology, 159, 415

[27] Nakamura, M., Nishida, T., Hikida, M. and Otori, T. (1994). Combined effects of hyaluronic acid and fibronectin on corneal epithelial wound closure of rabbit in vivo. Curr. Eye Res., 13, 385

[28] Nishida, T. (1993). Extra-cellular matrix and growth factors in corneal wound healing, Curr. Opin. Ophthal., 4(4), 4.

[29] Odland, G. F. (1983). Structure of the skin, in Biochemistry and Physiology of the Skin, L. A. Goldsmith (ed.), (Oup, Oxford), pp. 3-63. 
[30] Ohashi, Y., Morokura, M., et al. (1989). Presence of epidermal growth factor in human tears. Invest. Ophthalmol. Vis. Sci., 30, 1879-1882.

[31] Pastor, J. and Calonage, M. (1992). EGF and corneal wound healing: a multi-centre study. Cornea, 11, 311 .

[32] Schultz, G., Khaw, P., et al. (1994). Growth factors and ocular wound healing, Eye, $8,184$.

[33] Sharma, A. and Coles, W. (1989). Kinetics of corneal epithelial maintenance and graft loss. Investig. Ophthal. Vistual Science, 30, 1962.

[34] Sherratt, J. A. and Murray, J. D. (1990). Models of epidermal wound healing. Proc. R. Soc. Lond. B, 241, 29-36.

[35] Sherratt, J. A. and Murray, J. D. (1992). Epidermal wound healing: the clinical implications of a simple mathematical model. Cell Transplantation, 1, 365-371.

[36] Sherratt, J. A., Martin, P., Murray, J. D. and Lewis, J. (1992). Mathematical models of wound healing in empryonic and adult epidermis. IMA J. Math. Appl. Med. Biol., 9, 177-196.
[37] Sherratt, J. A. (1993). Act in aggregation and embryonic epidermal wound healing. J. Math. Biol, 31, 703-716.

[38] Sherratt, J. A. and Lewis, J. (1993). Stress-induced alignment of act in filaments and the mechanics of cytogel. Bull. Math. Biol., 55, 637-654.

[39] Steen, K. and Deplama, L. (1988). In Molecular and Cellular Biology of Wound Repair, R. Clark and P. Henson(eds), (Plenum, New York) pp. 321

[40] Thoft, F (1983). The XYZ hypothesis of corneal epithelial maintenence and graft loss. Investig. Ophthal. Visual Science, 24, 1442.

[41] Tseng, S. (1989). Concept and application of limbal stem cells., Eye, 3, 141.

[42] Watanabe, K., Nakagawa, S. and Nishida, T. (1987). Stimulatory effects of fibronectin and EGF on migration of the corneal epithelial cell. Investig. Ophthal. Visual Science, $\mathbf{2 8}$, 205.

[43] Wolfe, S. (1995). Introduction to Cell and Molecular Biology, Wadsworth Publishers Limited, California. 


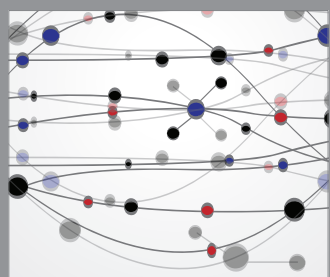

The Scientific World Journal
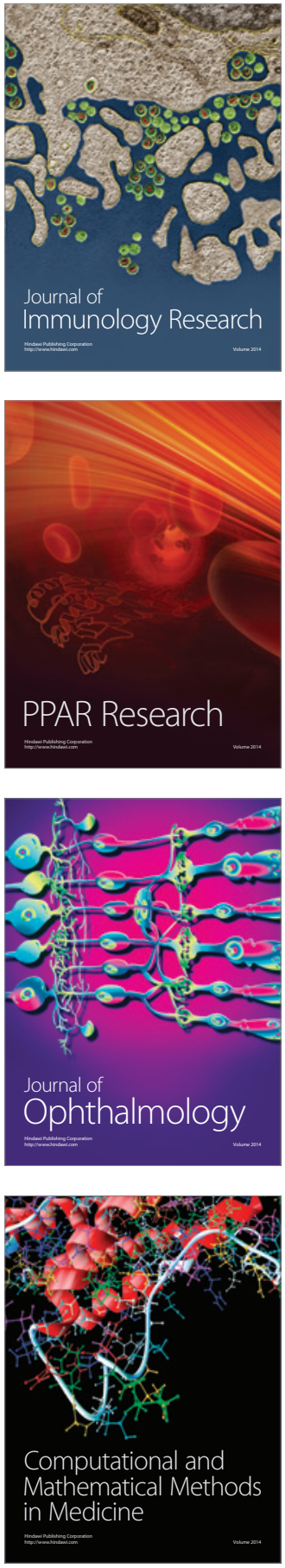

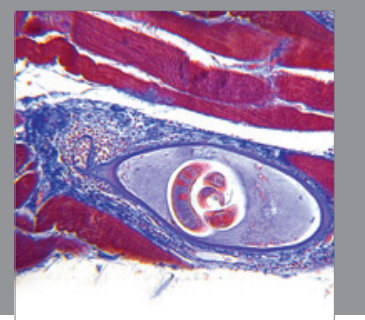

Gastroenterology

Research and Practice
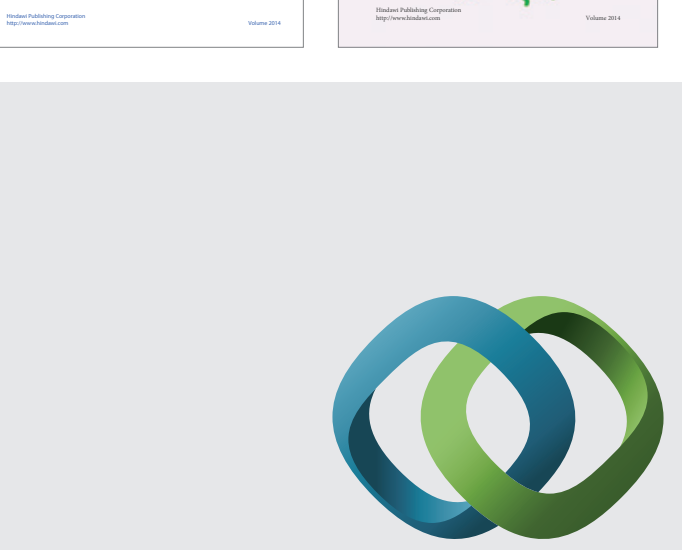

\section{Hindawi}

Submit your manuscripts at

http://www.hindawi.com
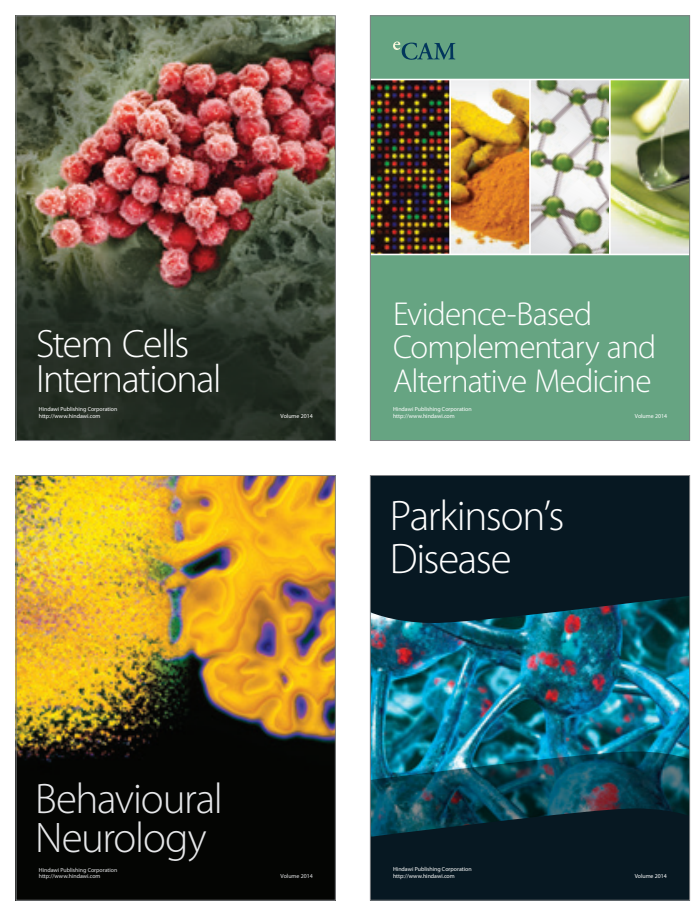

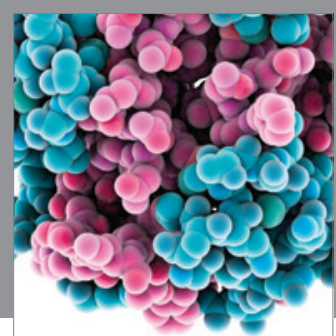

Journal of
Diabetes Research

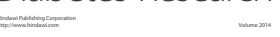

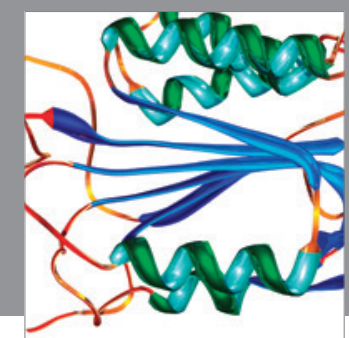

Disease Markers
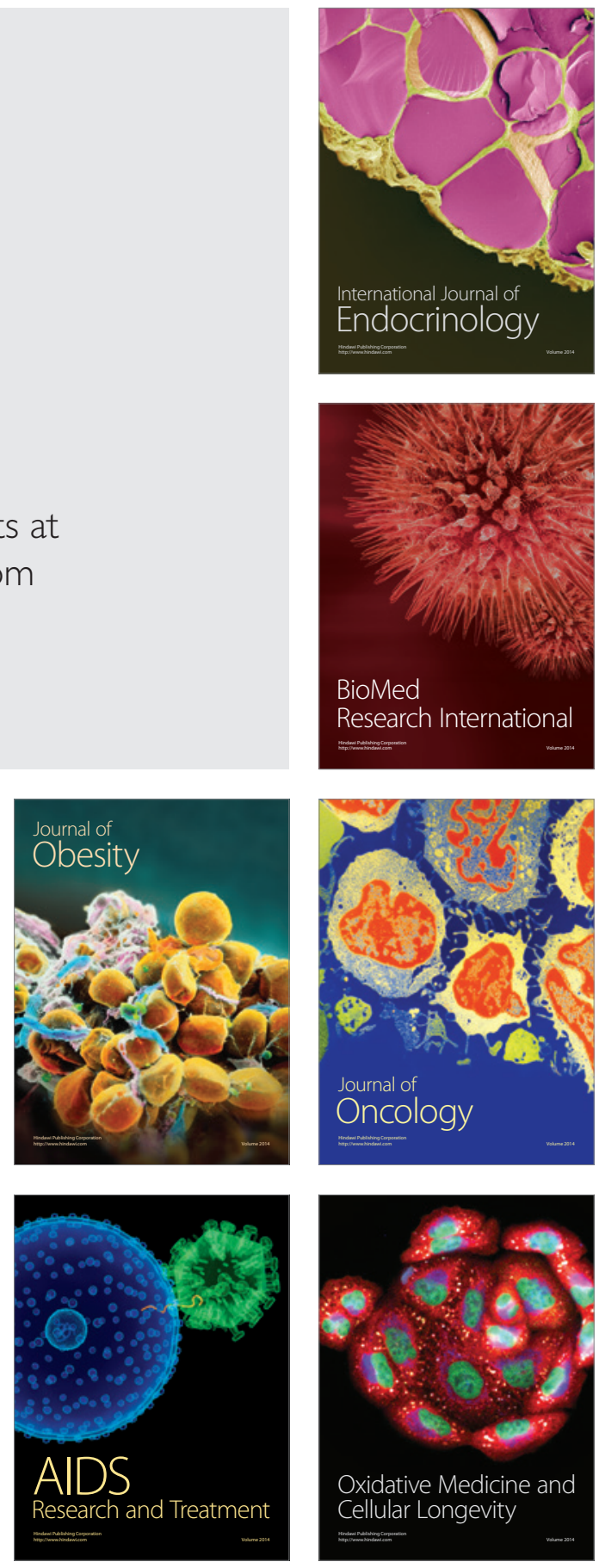